Monatsschr Kinderheilkd 2018 · 166:977-986 https://doi.org/10.1007/s00112-018-0545-8 Online publiziert: 27. Juli 2018

(c) Der/die Autor(en) 2018

Redaktion

S. Burdach, München

CrossMark

\author{
J. Skokowa $\cdot$ C. Zeidler ${ }^{2} \cdot$ K. Welte ${ }^{3}$ \\ ${ }^{1}$ Abteilung Onkologie, Hämatologie, Klinische Immunologie, Rheumatologie und Pulmologie, \\ Universitätsklinikum Tübingen, Tübingen, Deutschland \\ ${ }^{2}$ Klinik für Hämatologie, Hämostaseologie, Onkologie und Stammzelltransplantation, Medizinische \\ Hochschule Hannover, Hannover, Deutschland \\ ${ }^{3}$ Pädiatrische Hämatologie und Onkologie, Kinderklinik, Universitätsklinikum Tübingen, Tübingen, \\ Deutschland
}

\title{
Chronische Neutropenien im Kindesalter
}

einen Ausreifungsarrest auf der Stufe der Promyelozyten [23, 28]. Die Zahlen der eosinophilen Granulozyten und der Monozyten sind meist erhöht. Die Erythround Megakaryo-/Thrombozytopoese sowie die Lymphopoese verlaufen normal. Geht die Erkrankung mit der Zerstörung der gebildeten Granulozyten einher, wie z. B. bei AIN [3], findet sich im Knochenmark eine normale Ausreifung bis $\mathrm{zu}$ den stabkernigen neutrophilen Granulozyten. Patienten mit CN leiden schon in den ersten Lebensjahren an häufigen teilweise schweren bakteriellen Infektionen, v. a. des Mundbereichs (• Abb. 1), der Ohren, der Haut, der oberen Luftwege, der Lungen sowie seltener an Abszessen der Leber und Nieren [26, 28].

Die Verfügbarkeit des Wachstumsfaktors Granulozyten-koloniestimulierender Faktor (G-CSF; Filgrastim/ Lenograstim) für die Behandlung der Patienten seit 1987 [24] hat die Infektionshäufigkeit signifikant reduziert und damit die Lebensqualität und die Langzeitprognose der Betroffenen entscheidend verbessert. Während vor 1987 die Mehrzahl der Patienten an bakteriellen Komplikationen verstarb [10], ist heute die Lebenserwartung in der Mehrzahl der Patienten normal [23]. Durch die signifikante Verbesserung der Lebenserwartung hat ein Großteil der Patienten bereits das Erwachsenenalter erreicht und in Einzelfällen auch mit eigener Familienplanung begonnen. Einem „normalen“ Leben des Patienten und seiner Familie steht bei gutem Therapieansprechen, guter Therapieeinstellung und dem verantwortungsbewussten Umgang mit der Erkrankung kaum etwas im Wege. So sind z. B. Auslandsaufenthalte, etwa im Rahmen einer Urlaubsreise, nach Rücksprache mit dem behandelnden Arzt und bei vernünftiger Planung jeder Zeit möglich.

Angeborene Formen der SCN gehören zu den seltenen Erkrankungen mit

(SCN)“ beschreibt eine Gruppe unterschiedlicher, oft vererbter Erkrankungen der Blutbildung, die durch einen Mangel an neutrophilen Granulozyten im Blut mit absoluten Werten von weniger als $0,5 \cdot 10^{9} / 1$ charakterisiert ist $[5,23,26]$. Ursachen einer schweren Neutropenie können einerseits ein Defekt in der Proliferation und Differenzierung der neutrophilen Granulozytenvorstufen im Knochenmark, andererseits eine Zerstörung der gebildeten Granulozyten im Blut sein. Bei Erkrankungen, die mit einem Defekt in der Differenzierung der Granulozytenvorstufen einhergehen, wie z.B. bei $\mathrm{CN}$, zeigt das Knochenmark meist

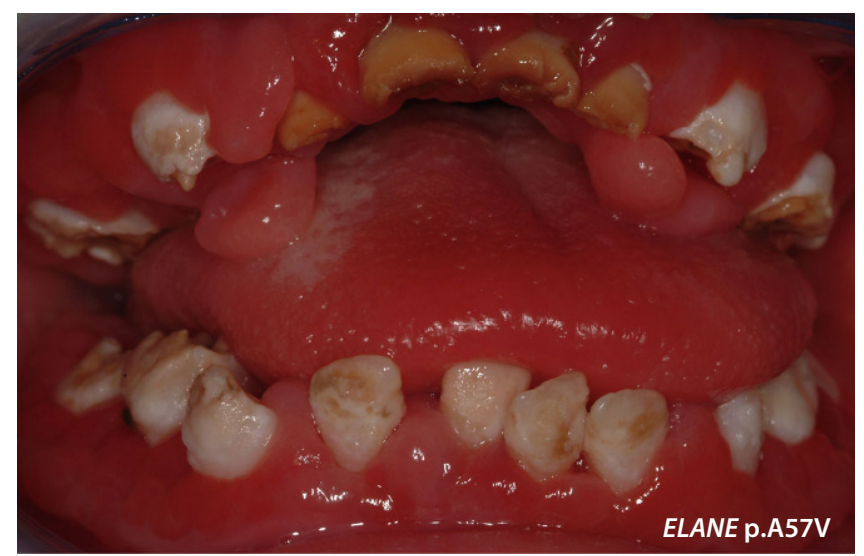

Abb. $1<$ Schwere Gingivitis einer 21 $\frac{1}{2}$ Jahre alten Patientin mitschwerer chronischer Neutropenie; kein Ansprechen auf Therapie mithilfe des Granulozytenkoloniestimulierenden Faktors 


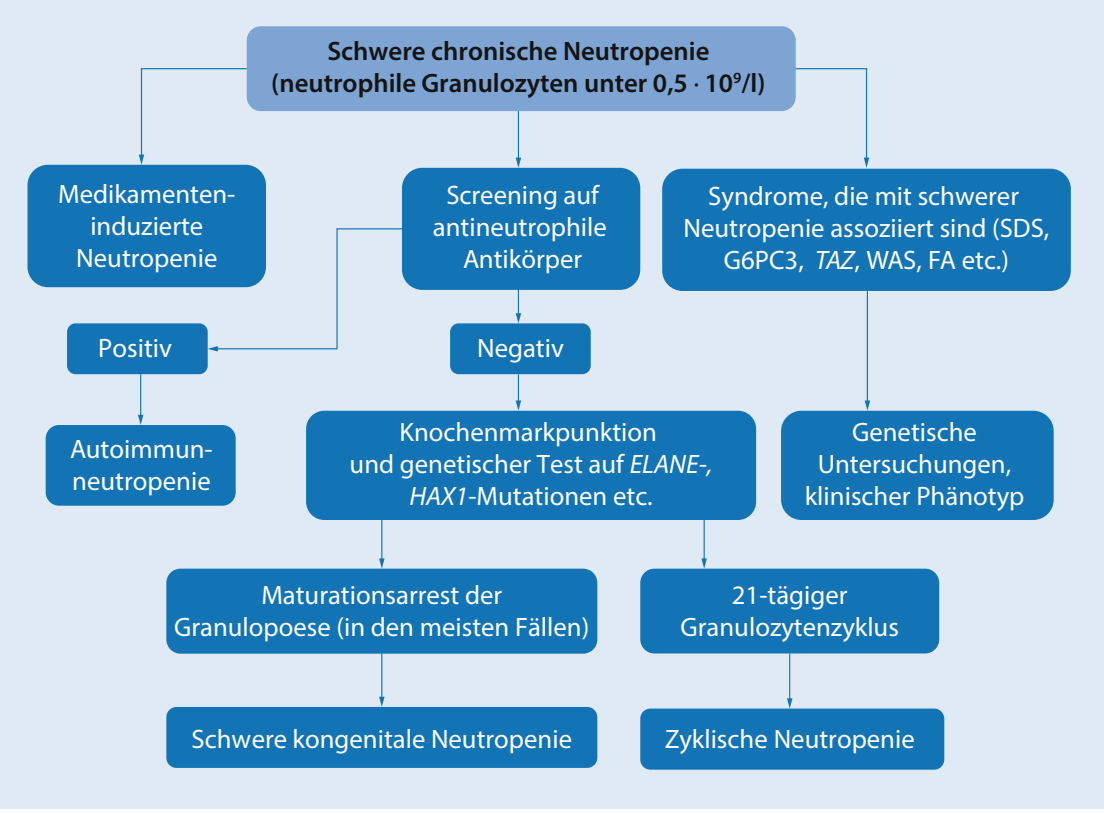

Abb. 2 ム Algorithmen zur Diagnostik von Patienten mit schwerer chronischer Neutropenie. FA Fanconi-Anämie, G6PC3 G6PC3-Defizienz, SDS Shwachman-Diamond-Syndrom, TAZ (Tafazzin) Barth-Syndrome, WAS Wiskott-Aldrich-Syndrom

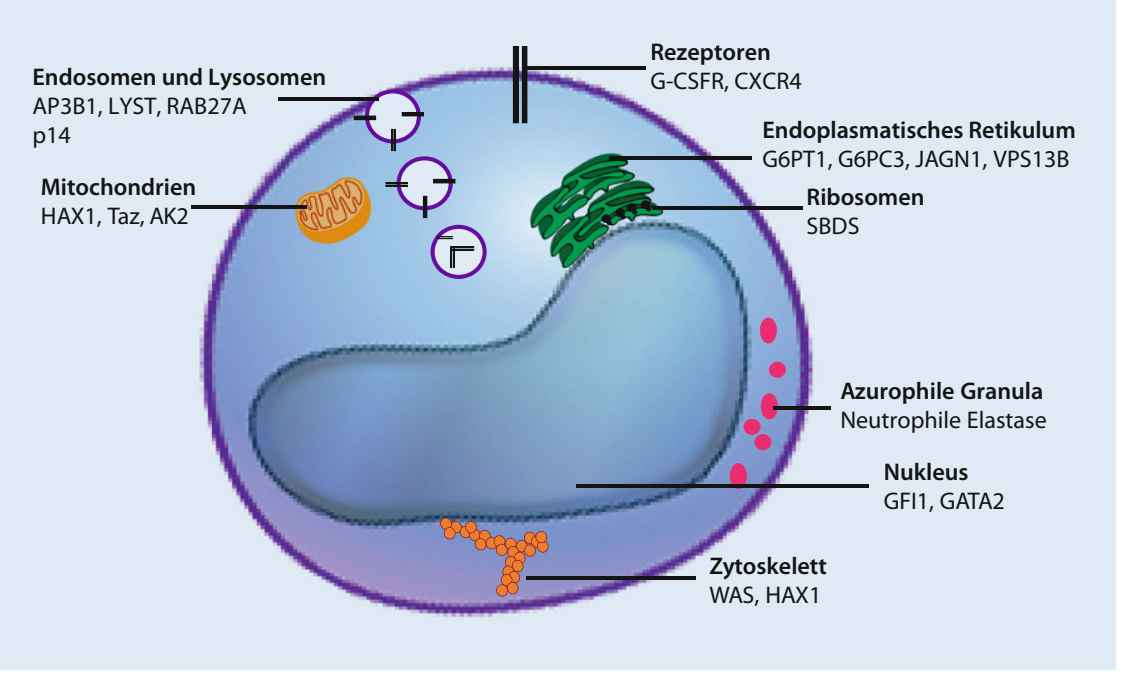

Abb. 3 ॥ Schematische Darstellung der zellulären Lokalisation der Proteine, die bei Patienten mit schwerer chronischer Neutropenie mutiert werden können

einer Häufigkeit von ca. 2 bis 4 Patienten auf 1 Mio. Einwohner [23]. Aufgrund der geringen Inzidenz der einzelnen Erkrankungen sind die heutigen Erkenntnisse zu Pathophysiologie und klinischem Verlauf v.a. dem Aufbau eines internationalen Erkrankungsregisters für Neutropenie zu verdanken [5]. Seit 1994 sammelt das „Severe Chronic Neutropenia International Registry" (SCNIR; Kodirektoren: Dr. David Dale und Dr. Karl Welte) mit Sitzen in der University of Washington,
Seattle, USA, sowie der Medizinischen Hochschule Hannover und dem Universitätsklinikum Tübingen weltweit Longitudinaldaten von inzwischen mehr als 3000 Patienten mit angeborenen und erworbenen SCN. Erfasst werden Erkrankungsverlauf, sekundäre Erkrankungen, Therapieansprechen und Nebenwirkungen der Therapie (www.scnir.de).

Im Zuge der Lebensverlängerung unter einer Langzeittherapie mit dem hämatopoetischen Wachstumsfaktor G-CSF demaskierte sich allerdings auch das erhöhte Risiko einer malignen Transformation, eines Übergangs in ein myelodysplastisches Syndrom (MDS) oder eine akute myeloische Leukämie (AML; [23]).

\section{) Seit 1994 sammelt SCNIR weltweit Longitudinaldaten von inzwischen mehr als 3000 SCN- Patienten}

Moderne molekularbiologische Techniken wie die Sequenzierung der Gene, die für die Entstehung der Neutropenie verantwortlich sind, und die Grundlagenforschung haben in den letzten Jahren neue Einblicke in die Mechanismen der Erkrankungsentstehung und malignen Transformation ermöglicht. Die Identifikation von Indexfamilien innerhalb des SCNIR führte zur Aufdeckung neuer genetischer Defekte $[6,7,12,13]$. Die enge Kooperation zwischen Patienten, klinischen Experten und Wissenschaftlern im SCNIR-Netzwerk ermöglicht die gezielte Suche nach charakteristischen klinischen Merkmalen und Besonderheiten in den genetischen Untergruppen (Genotyp-Phänotyp-Analyse).

\section{Diagnose}

Die normale Zahl der peripheren neutrophilen Granulozyten unterliegt im Kindesalter erheblichen Schwankungen, während die Werte im Erwachsenenalter weitgehend stabil sind. Bei wiederholt gemessenen Granulozytenwerten unterhalb $0,5 \cdot 10^{9} / 1 \mathrm{im}$ Blut liegt eine SCN vor. Sie kann isoliert oder in Verbindung mit weiteren Symptomen, wie Verminderung anderer Blutzellreihen, aber auch Organfehlbildungen, Gedeihstörung, Stoffwechselstörungen und Immunphänomenen auftreten.

Die neutropenieassoziierten Krankheitssymptome hängen bei der Mehrzahl der Neutropenieformen vom Schweregrad und von der Dauer der Neutropenie ab. Je niedriger die Neutrophilenzahl ist, desto höher ist das Risiko für eine Infektion, wenn die Neutropenie länger als 3 Tage andauert. Beim Fehlen der neu- 
trophilen Granulozyten verlaufen diese Infektionen trotz Antibiotikatherapie oft rasch progredient und manchmal auch letal.

Ausreichende Hinweise zur Einteilung einer vorliegenden Neutropenie gibt häufig bereits das folgende diagnostische Vorgehen:

- klinische Untersuchung mit Suche nach weiteren organischen Auffälligkeiten,

- Familienanamnese mit der Frage nach weiteren Betroffenen und Verwandtenehen,

- laborchemische Suche nach antigranulozytären Antikörpern in einem spezialisierten Labor [3],

- Untersuchung des Knochenmarks zur Identifizierung einer Reifungsstörung mit zytogenetischer Analyse und zum Ausschluss einer bereits vorliegenden malignen Transformation [22] sowie

- immunologische Diagnostik zum Ausschluss primärer lymphatischer Immundefekterkrankungen.

Bei Verdacht auf eine CN sind die molekulargenetische Analyse der über 20 bisher bekannten Gene [23] und die Suche nach noch nicht bekannten Genmutationen indiziert. Neben der Identifizierung der molekularen Ursachen der Erkrankung ergeben sich bei Nachweis einer Genmutation wichtige Hinweise für die Langzeitprognose des Patienten. Die Algorithmen zur Diagnostik sind in - Abb. 2 dargestellt.

\section{Mortalität}

Die Mortalität der schweren angeborenen Neutropenie (CN) vor der Antibiotikaära betrug $90 \%$ (vor 1950; [10]). Die Verfügbarkeit von Antibiotika in den Jahren zwischen 1950 und 1990 konnte die Sterblichkeit lediglich auf etwa $80 \%$ senken. Der eigentliche Durchbruch in der erfolgreichen Behandlung der CN kam in den Jahren nach 1990, als der Wachstumsfaktor der Granulozyten (G-CSF, Filgrastim/Lenograstim [24]) therapeutisch erhältlich wurde. Heute überleben mehr als $90 \%$ aller Patienten.

Monatsschr Kinderheilkd 2018 · 166:977-986 https://doi.org/10.1007/s00112-018-0545-8 (c) Der/die Autor(en) 2018

\section{J. Skokowa $\cdot$ C. Zeidler $\cdot$ K. Welte \\ Chronische Neutropenien im Kindesalter}

\section{Zusammenfassung}

Die schwere chronische Neutropenie (,severe chronic neutropenia", SCN) umfasst eine heterogene Gruppe von Erkrankungen mit einem gemeinsamen hämatologischen und klinischen Phänotyp, der durch eine absolute Neutrophilenzahl unterhalb $0,5 \cdot 10^{9} / \mathrm{l}$ und ein erhöhtes Risiko für schwere bakterielle Infektionen charakterisiert ist. Die Unterscheidung zwischen angeborener und erworbener Neutropenie sowie die Identifikation ursächlicher Genmutationen sind von immenser Bedeutung für die Bewertung der Prognose. In den ersten 3 bis 5 Lebensjahren sind die primären Autoimmunneutropenien die häufigste Ursache einer SCN. Angeborene, vererbte Neutropenien haben trotz ihrer Seltenheit einen erheblichen Wert für die Erforschung normaler und pathologischer Blutbildung sowie eine fundamentale Bedeutung für das heutige Verständnis der
Hämatopoese. Bisher konnten mehr als 30 verschiedene Genmutationen beschrieben werden, die mehrheitlich mit einem erhöhten Leukämierisiko (bis zu 20\%) assoziiert sind. Durch die Verfügbarkeit und Behandlung mit dem hämatopoetischen Wachstumsfaktor Granulozyten-koloniestimulierender Faktor (G-CSF; Filgrastim/Lenograstim) seit 1987 hat sich die Langzeitprognose von SCN-Patienten dramatisch verbessert: Bakterielle Infektionen werden verhindert, und die Lebensqualität der Betroffenen hat sich normalisiert.

\section{Schlüsselwörter}

Syndrome der gestörten Blutbildung . Präleukämie · Granulozyten-koloniestimulierender Faktor - Angeborene Neutropenie . Internationales Register für schwere chronische Neutropenien

\section{Chronic neutropenia in childhood}

\section{Abstract}

Severe chronic neutropenia (SCN) comprises a heterogeneous group of disorders with a common hematological and clinical phenotype characterized by an absolute neutrophil count (ANC) below $0.5 \cdot 10^{9} /$. Patients with SCN are prone to recurrent, often life-threatening bacterial infections. The discrimination between congenital and acquired neutropenias and the identification of causative gene mutations is of great importance for estimation of the prognosis. In the first 3-5 years of life, primary autoimmune neutropenia is the most frequent cause of SCN. Despite their rarity, congenital inherited neutropenias are of great value for the research on normal and pathological hematopoiesis and have a fundamental impact on the current knowledge of hematopoiesis. To date mutations in more than 30 different genes have been described, which are mainly associated with an increased risk (approximately 20\%) for leukemia. Due to the availability and the treatment with the hematopoietic growth factor granulocyte colony-stimulating factor (GCSF, filgrastim/lenograstim) the long-term prognosis of SCN patients has significantly improved. Bacterial infections can be prevented and patients experience an almost normal quality of life.

\section{Keywords}

Bone marrow failure syndromes - Preleukemia. Granulocyte colony-stimulating factor Congenital neutropenia - Severe Chronic Neutropenia International Registry

\section{Angeborene Neutropenien}

Der Begriff „kongenitale Neutropenie“ beschreibt eine sehr heterogene Erkrankungsgruppe mit isolierten Blutbildungsstörungen und Neutropenien, die mit anderen Störungen in Form einer Syndromerkrankung assoziiert sind. Die genetischen Untergruppen in 777 europäischen
Patienten mit CN sind in $\bullet$ Tab. 1 aufgeführt.

Beispielhaft sind in 0 Infobox 1 die häufigsten genetischen Ursachen einer $\mathrm{CN}$ aufgelistet [23].

Die für die CN ursächlichen Genmutationen führen zu veränderten Proteinen in unterschiedlichen Kompartments der neutrophilen Granulozyten (-Abb. 3). 
Tab. 1 Genetische Untergruppen von 777 Patienten mit schwerer chronischer Neutropenie (Stand September 2017)

\begin{tabular}{|c|c|}
\hline Neutropeniesubtyp/Genmutation & Patienten \\
\hline Kongenitale Neutropenie (CN), gesamt & 462 \\
\hline ELANE & 122 \\
\hline HAX1 & 48 \\
\hline G6PC3 & 9 \\
\hline JAGN1 & 3 \\
\hline „Digenic mutations" & 5 \\
\hline CN, nicht klassifiziert & 117 \\
\hline Shwachman-Diamond-Syndrom & 95 \\
\hline - SBDS, nicht getestet & 16 \\
\hline - SBDS-pos. & 78 \\
\hline - SBDS-neg. & 1 \\
\hline Glykogenspeicherkrankheit Typ Ib (SLC37A4) & 30 \\
\hline $\begin{array}{l}\text { Myelokathexis, WHIM-Syndrom (CXCR4); Warzen-Hypogammaglobulinämie- } \\
\text { Immundefizienz-Myelokathexis(WHIM)-Syndrom (CXCR4-pos.) }\end{array}$ & 7 \\
\hline Barth-Syndrom (TAZ-pos.) & 6 \\
\hline WAS-Mutation & 7 \\
\hline p14 (LAMTOR2)-Mutation & 4 \\
\hline Kongenitale Aplasie von weißen Blutzellen & 1 \\
\hline Clericuzio type poikiloderma (USB1) & 2 \\
\hline Cohen Syndrom (VPS13B) & 4 \\
\hline Pearson-Syndrom & 1 \\
\hline Chediak-Higashi-Syndrom (LYST) & 1 \\
\hline Zyklische Neutropenie & 98 \\
\hline - ELANE-pos. & 56 \\
\hline - ELANE, getestet, negativ & 9 \\
\hline - ELANE, nichtgetestet & 33 \\
\hline Idiopathische Neutropenie & 85 \\
\hline Autoimmunneutropenie & 107 \\
\hline Andere Formen & 25 \\
\hline - Hyper lgM Syndrom (CD4OLG) & 4 \\
\hline - „Large-granular-lymphocyte“(LGL)-Leukämie & 5 \\
\hline - Diagnose unklar & 16 \\
\hline
\end{tabular}

\section{Vererbung}

Autosomal-dominant vererbte Neutropenien wie z.B. ELANE-CN $[6,15]$ sind insgesamt sehr viel häufiger als autosomal-rezessiv vererbte Neutropenien (z. B. HAX1-CN; [13]), die v. a. in konsanguinen Familien diagnostiziert werden. Die Prävalenz der durch Mutationen in HAX1 verursachten kongenitalen Neutropenien in Familien türkischer und arabischer Abstammung in Deutschland ist durch die bekannte Konsanguinität in vielen dieser Familien erklärbar. Interessanterweise ist die früher als „Kostmann-Syndrom" bezeichnete $\mathrm{CN}$ erstmals in kon- der Sequenzierung des HAX1-Gens oder den entsprechenden anderen Mutationen (s. unten) begonnen werden. Aus Afrika stammende Patienten weisen häufig spezifische Polymorphismen im DARC-Gen auf (Duffy-Antigen; [23]). Das Referenzlabor des SCNIR in Tübingen koordiniert die Gendiagnostik bei Patienten mit Verdacht auf $\mathrm{CN}$; auch erworbene Mutationen im CSF3R- und in leukämieassoziierter Genen werden analysiert (Kontakt: julia.skokowa@med.uni-tuebingen.de).

\section{Phänotypen}

Angeborene Neutropenien z. B. mit $\mathrm{Mu}$ tationen der Gene ELANE oder HAX1, lassen sich aufgrund klinischer Merkmale oder anhand der Knochenmarkmorphologie nicht unterscheiden [28]: Im Blutbild fehlen neutrophile Granulozyten häufig vollständig; zusätzlich können eine milde Anämie (Hämoglobinwert $10-12 \mathrm{~g} / \mathrm{dl}$ ) und Thrombozytose (bis ca. $800.000 / \mu \mathrm{l}$ ) bestehen. Die chronische Neutropenie wird durch wiederholte Differenzialblutbilder bestätigt. Im Knochenmark zeigt sich typischerweise ein "Ausreifungsstopp“ auf einer frühen Vorstufe der Myelopoese (Stadium der Promyelozyten-/Myelozyten). Die Promyelozyten weisen Veränderungen wie Vakuolisierung oder Kernatypien auf ([23, 26]; - Abb. 4). Eine Knochenmarkeosinophilie findet sich häufig. Zahl und morphologische Eigenschaften der Megakaryozyten sind normal. Eine Spiegelerhöhung des Immunglobulin(Ig)G findet sich bei der Mehrzahl der Patienten, unabhängig von ihrem Infektionsstatus. Die spezifische Immunkompetenz nach einer Impfung ist normal. Ohne Behandlung erleiden die Patienten schwere bakterielle Infektionen, wie z. B. Omphalitis, Bronchitiden, Pneumonien, Hautabszesse oder Otitis media - häufig bereits während der ersten Lebensmonate. Daher wird bei den meisten Patienten die Diagnose schon in der frühen Kindheit gestellt.

Differenzialdiagnostisch ist die schwere $\mathrm{CN}$ [26] von der Immunneutropenie [3] dadurch abzugrenzen, dass bei $\mathrm{CN}$ die Reifungsstufen nach den Promyelozyten/Myelozyten fast völlig fehlen, während bei der Immunneutropenie alle 


\section{Infobox 1 Erkrankungen der Hämatopoese \\ Primäre Autoimmun- neutropenie}

1. Isolierte Neutropenien

- ELANE-CN

- ELANE-CyN: zyklische Neutropenie

- HAX1-CN: Kostmann-Syndrom

- CXCR2-CN: Myelokathexis

- GFI1-CN

2. Neutropenie, assoziiert mit Stoffwechselerkrankungen

- SLC37A4-CN: Glykogenose Typ 1b

- SBDS-CN: exokrine Pankreasinsuffizienz (Shwachman-Diamond-Syndrom)

- TCN2-CN: "transcobalamin deficiency“

3. Neutropenie, assoziiert mit Immundefekterkrankungen

- CD40LG-CN: Hyper-lgM-Syndrom

- RAB27A-CN: partieller Albinismus und Immundefekt (Griscelli-Syndrom)

4. Neutropenie, assoziiert mit syndromalen Erkrankungen

- G6PC3-CN: kardiale und urogenitale Fehlbildungen

- LYST-CN: okulokutaner Albinismus und Hepatosplenomegalie (Chediak-Higashi-Syndrom)

- TAZ-CN: Kardiomyopathie (Barth-Syndrom)

- JAGN1-CN: Knochen- und Zahndefekte

5. Neutropenien im Rahmen anderer hämatologischer Erkrankungen

- angeborene Störungen mit Knochenmarkversagen (z. B. Fanconi-Anämie)

Vorstufen der neutrophilen Granulopoese bis zu den stabkernigen Granulozyten vorhanden sind.

Die zyklische Neutropenie ist eine Sonderform der CN. Bei der Mehrzahl der Betroffenen (ca. 90\%) werden ebenfalls autosomal-dominant vererbte Mutationen im ELANE-Gen gefunden $[6,12,15]$. Anstieg und Abfall der Neutrophilenzahlen folgen dem konstanten Zyklus von etwa 21 Tagen [23]. In der Zyklustalphase kommt es zu einer schweren Neutropenie, die bei manchen Patienten bis zu 8 Tagen andauert. Bei anderen Patienten hält die schwere Neutropenie während des gesamten Zyklus an. Die Häufigkeit bakterieller Infektionen steigt mit der Dauer dieser Neutropenieperioden an. Die Diagnose wird anhand der Dokumentation des neutrophilen Verlaufs mithilfe von Differenzialblutbildern 3-mal/Woche über 6 Wochen gestellt.
Die AIN ist die häufigste Ursache einer SCN im Kindesalter und tritt nicht im Gefolge einer bereits bestehenden Grunderkrankung auf. Häufig fällt die Neutropenie im Rahmen von Routineblutuntersuchungen auf [3]. Das Patientenalter bei Diagnosestellung beträgt im Median 7 Monate, daher der Name „Autoimmunneutropenie im Kleinkindalter". Im Unterschied zu den Patienten mit $\mathrm{CN}$ leiden die Betroffenen trotz der schweren Neutropenie häufig nur unter leichten bakteriellen Infektionen wie Gingivitis oder Otitis media. Die Neutrophilenzahlen können im Infektionsstadium ansteigen [3]. Die Autoantikörper richten sich v.a. gegen Antigene auf dem $F_{c \gamma}$-Rezeptor IIIb (CD16b). Für den Antikörpernachweis ist die kombinierte Durchführung verschiedener Testmethoden wie Granulocyte Agglutination Test (GAT), Granulocyte Immunfluorescence Test (GIFT), Lymphocyte Immunfluorescence Test (LIFT) und Monoclonal Antibody Immobilization of Granulocyte Antigens (MAIGA) erforderlich [3]. Bei den meisten Patienten normalisiert sich die Zahl der neutrophilen Granulozyten bis zum Eintritt in die Schule spontan. Eine Behandlung ist nicht erforderlich. Patienten mit rezidivierenden leichteren bakteriellen Infektionen können jedoch von einer Antibiotikaprophylaxe, insbesondere über die Wintermonate, profitieren. Eine G-CSF-Therapie sollte nur in Ausnahmefällen bei vorliegenden schweren Infektionen eingeleitet werden.

\section{Sekundäre Neutropenien}

Sekundäre Neutropenien basieren auf bereits bestehenden Erkrankungen und Einflüssen. Sie sind dementsprechend zahlreich und vielfältig [1]. Im Folgenden werden die wesentlichen Störungen gelistet:

- Sekundäre Autoimmunneutropenie im Rahmen weiterer Autoimmunerkrankungen z. B. erythematodes, rheumatische Arthritis;

- infektionsbedingte Neutropenie (Grippe-, Masern-, Rötelnvirus,
Parvovirus B19 [Ringelröteln], Varizellen, Hepatitis-, Epstein-Barr-, humanes Immundefizienzvirus [HIV]; bakterielle Sepsis; Malaria); - idiosynkratische Medikamentenreaktionen können eine schwere Neutropenie verursachen (Frequenz ca. 12 Fälle/1 Mio. Einwohner). Zwei ursächlich unterschiedliche Typen kommen vor: Eine Ursache ist die dosisabhängige Interferenz mit der Proteinsynthese und Zellproliferation. Prototypen dafür sind Neutropenien, induziert durch Metamizol, Phenothiazine, Thyreostatika, Chloramphenicol und Clozapin. Der zweite Typ ist dosisunabhängig; die Neutropenie ist hier eher immunologischen Ursprungs;

- zytostatikainduzierte Neutropenie (im Rahmen einer Krebstherapie);

- Neutropenie bei Malnutrition (z. B. Vitamin- $B_{12}$, Folsäure-, Kupfer-, Eisenmangel);

- idiopathische Neutropenie;

- Neutropenien im Rahmen von anderen erworbenen hämatologischen Erkrankungen, wie erworbene Störungen mit Knochenmarkversagen (z. B. aplastische Anämie) oder Leukämien.

\section{Pathogenese}

Die Pathomechanismen, die der CN zugrunde liegen bzw. die Ausreifung der Vorstufen der neutrophilen Granulozyten verhindern, sind je nach genetischer Ursache der Neutropenie unterschiedlich. „CCAAT/enhancer-binding protein alpha“ (C/EBPalpha) und „lymphoid enhancer-binding factor 1 " (LEF1) sind nach Stimulation mit G-CSF aktivierte hämatopoetisch spezifische Transkriptionsfaktoren, die nach Bindung an die entsprechenden Gene die Differenzierung von myeloischen Vorläuferzellen in reife neutrophile Granulozyten stimulieren. Ihre deregulierte Expression [4, $18,19]$ ist eine gemeinsame Ursache der fehlenden Differenzierung von Vorläuferzellen zu reifen neutrophilen Granulozyten. ELANE-Mutationen resultieren in falsch gefalteten Elastaseproteinen, die nicht normal abgebaut werden können, im endoplasmatischen Retikulum (ER) 


\section{Leitthema}

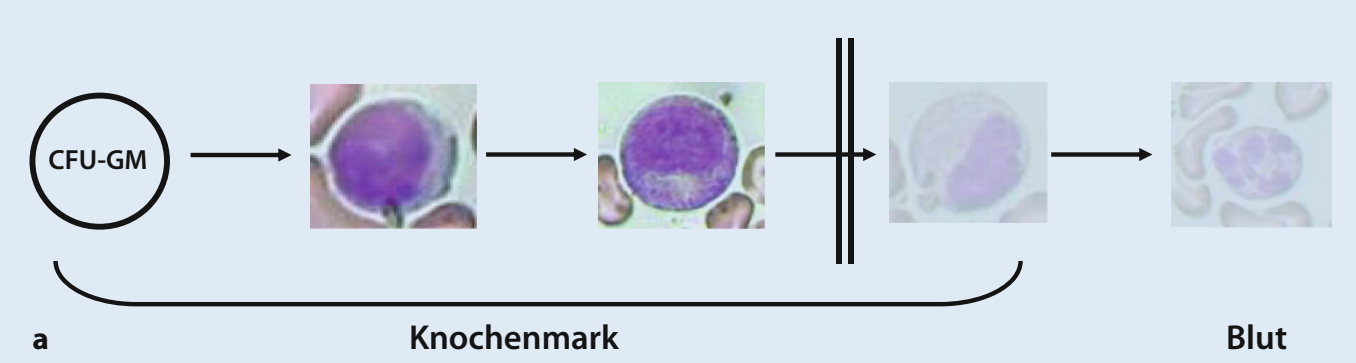

Knochenmark

Blut

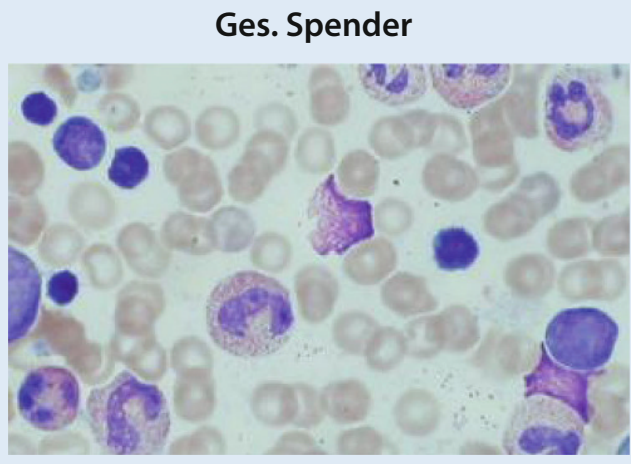

b
ELANE-CN p.C151Y

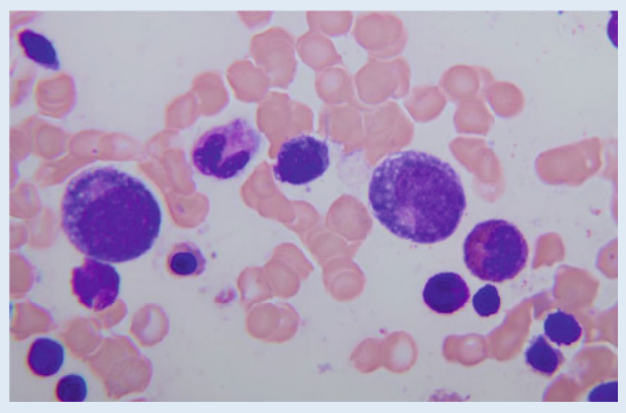

c
Abb. $4 \triangleleft$ Knochenmarkmorphologie bei SCN-Patienten. a Schematische Darstellung des Ausreifungsstopps der Granulopoese der Knochenmarkstammzellen auf der Stufe von Promyelozyten. CFU-GM "colony forming unit-granulocyte macrophage"; b,c HE-gefärbte Knochenmarkausstriche eines gesunden Spenders (b) und eines Patienten mit ELANE-CN (c). (Vergr. 400:1)

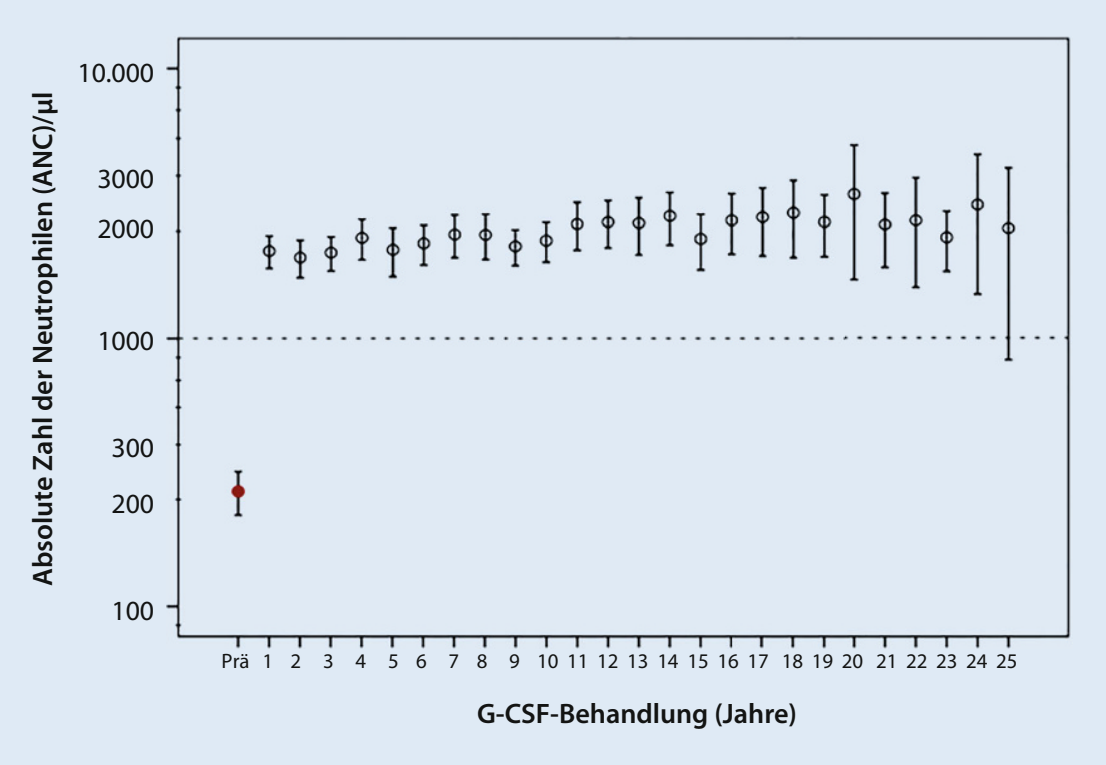

Abb. 5 A Absolute Zahl der neutrophilen Granulozyten bei Patienten mit schwerer chronischer Neutropenie (SCN) unter der Behandlung mit Granulozyten-koloniestimulierendem Faktor (G-CSF); SCN-Patienten ohne (roter Zirkel) und mit (weißer Zirkel) G-CSF-Therapie sind dargestellt

akkumulieren und dadurch Stress im ER und sekundär Apoptose verursachen [16]. HAX1-Mutationen führen zum Verlust mitochondrialer Funktionen in granulozytären Zellen [13], hemmen aber auch die G-CSF-Rezeptor-abhängigen Signalwege via "haematopoetic cell specific Lyn substrate 1“ (HCLS1, [21]). Ein interessanter Forschungsaspekt der eigenen Arbeitsgruppe ist, dass die Expression des Enzyms Nicotinamidphosphoribosyltransferase (NAMPT) bei Patienten mit angeborenen Neutropenien sehr stark erhöht ist [20]. Nicotina- midphosphoribosyltransferase bewirkt eine Erhöhung auch des intrazellularen $\mathrm{NAD}^{+}$-Gehalt (oxidierte Form des Nicotinamidadenindinukleotids) und sekundär zur Aktivierung der Deacetylase Sirtuin-1 (Sirt1). Die Daten zeigen, dass über diesen Mechanismus die GCSF-Produktion und die Erhöhung der Expression der GCSF-Rezeptoren induziert werden, sodass eine Therapie mit dem Substrat von NAMPT, nämlich mit Vitamin $B_{3}$ (Nikotinsäureamid), bei Patienten mit einem milden Phänotyp die Neutropenie korrigieren kann [20].

\section{Therapie}

In klinischen Studien konnte gezeigt werden, dass bei mehr als $90 \%$ der Patienten mit schwerer $\mathrm{CN}$ individuell unterschiedliche pharmakologische G-CSF-Dosen (1-80 $\mu \mathrm{g} / \mathrm{kgKG}$ und Tag s.c. appliziert) einen signifikanten und dauerhaften Anstieg der absoluten Neutrophilenzahl auf Werte über $1000 / \mu l$ induzieren (• Abb. 5; [2, 5, 25]). Hierdurch reduziert sich auch die Zahl schwerer bakterieller Infektionen signifikant. Die Therapie mit G-CSF wird von der Mehrzahl der Patienten gut 


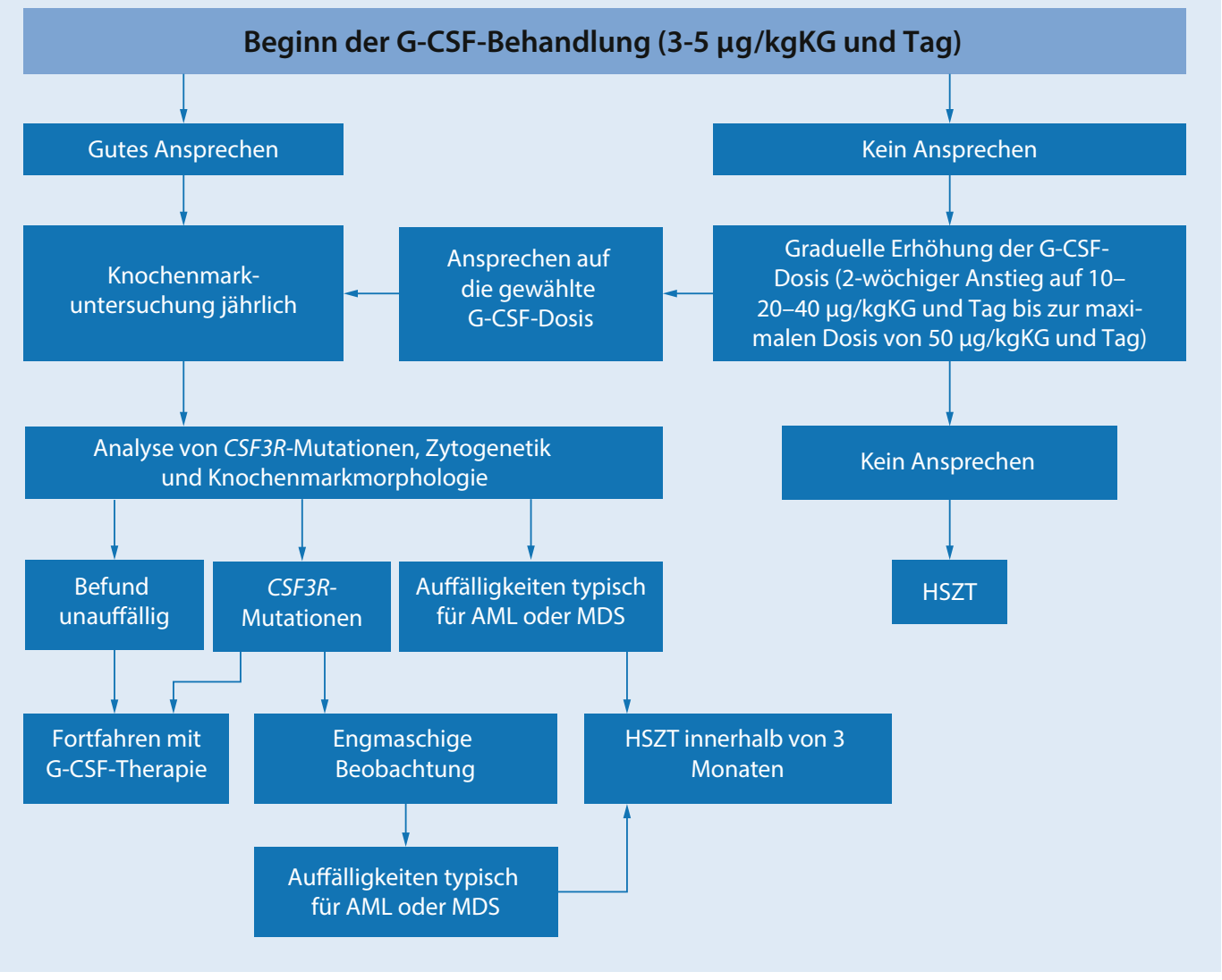

Abb. $6 \triangleleft$ Algorithmen der Anpassung der Therapie mit Granulozyten-koloniestimulierendem Faktor (G-CSF) bzw. der G-CSF-Dosis bei Patienten mit schwerer chronischer Neutropenie. $A M L$ akute myeloische Leukämie, MDS myelodysplastisches Syndrom, HSZT hämatopoetische Stammzelltransplantation

vertragen. Bei guter Therapieeinstellung und verantwortungsbewusstem Umgang mit einer SCN steht einem "normalen“ Leben des Patienten und seiner Familie kaum etwas im Wege.

Die G-CSF-Behandlung wird initial mit einer Dosis von $3-5 \mu \mathrm{g} / \mathrm{kgKG}$ und Tag s.c. begonnen; bei Erreichung von neutrophilen Granulozytenzahlen über $1000 / \mu \mathrm{l}$ Blut wird diese Dosis weitergeführt. Betragen die Neutrophilenzahlen weiterhin unter $1000 / \mu l$, werden die G-CSF-Dosen 2-wöchentlich erhöht (10, 20,40 etc. $\mu \mathrm{g} / \mathrm{kgKG}$ und Tag), bis über 1000 Granulozyten/ $\mu$ l erreicht sind und dann die Behandlung mit der entsprechenden Dosis weitergeführt wird ([2, 23, 25, 26]; - Abb. 6).

Auch Patienten mit einer zyklischen Neutropenie profitieren von einer G-CSF-Therapie. Diese Patienten sprechen schon auf Dosen von 1-5 $\mu \mathrm{g} / \mathrm{kgKG}$ und Tag mit einem signifikanten Zahlenanstieg der neutrophilen Granulozyten an. Allerdings wird der zyklische Verlauf der Neutrophilenzahlen durch die Gabe von G-CSF nicht auf-, sondern nur auf ein höheres Niveau mit einer verkürzten Neutropeniephase angehoben [23]. Ziel der G-CSF-Therapie ist die Verkürzung des Nadirs auf einen bis 3 Tage. Darunter treten schwere bakterielle Infektionen, aber auch Stomatitiden und Aphthen nicht mehr auf.

\section{》) Gute Einstellung der G-CSF- Therapie erlaubt der Mehrzahl der CN-Patienten ein "normales" Leben}

Bei Patienten, die nicht auf G-CSF ansprechen oder eine Leukämie entwickeln, muss eine Knochenmarktransplantation durchgeführt werden [27].

Patienten mit einer AIN werden, wie bereits beschrieben, nur bei schweren bakteriellen Infektionen kurzzeitig mit G-CSF behandelt. Die meisten dieser Kinder werden nur überwacht. Je nach Häufigkeit und Art der Infektion kann eine prophylaktische Therapie z.B. mit Cotrimoxazol hilfreich sein [3].
Alle anderen Formen der chronischen Neutropenie erfordern die Anpassung der Therapieempfehlungen an den individuellen Krankheitsverlauf. Bei medikamenteninduzierter Neutropenie führt das Absetzen des Medikaments meist nach ca. einer Woche zur Heilung. Auch hier kann im Fall von schweren bakteriellen Infektionen die G-CSF-Gabe therapeutisch von Nutzen sein.

\section{Leukämie}

Die CN ist schon in den 1970er-Jahren als präleukämisches Syndrom beschrieben worden [10]. Die statistische Auswertung von Langzeitverläufen bei 374 Patienten mit SCN (1987-2000) unter G-CSFLangzeittherapie durch das SCNIR identifizierte erstmals das statistische Leukämierisiko dieser Patientengruppe [17]. Nach 10 Jahren betrug die kumulative Inzidenz $21 \%$ für MDS/AML. Interessanterweise zeigte sich auch ein Einfluss der für einen Granulozytenzahlanstieg benötigten G-CSF-Dosis auf die Leukämiehäufigkeit als Hinweis darauf, 
Tab. 2 Inzidenz von sekundären Leukämien bei 574 Patienten mit chronischer Neutropenie (Stand September 2017)

\begin{tabular}{|l|l|l|}
\hline Diagnose & Anzahl (n) der Patienten & MDS/Leukämie ( [\%]) \\
\hline Kongenitale Neutropenie & 381 (ohne SDS) & $43(11,3)$ \\
\hline - ELANE-CN & 122 & $17(13,93)$ \\
\hline - HAX1-CN & 48 & $7(14,6)$ \\
- WAS-CN & 21 & $2(9,52)$ \\
\hline - Glykogenose Typ 1b (G6PT-pos.) & 30 & $1(3,33)$ \\
\hline - JAGN1-CN & 3 & $1(33,3)$ \\
\hline - Andere (G6PC3, TAZ, CXCR4 etc.) & 40 & 0 \\
\hline - Nichtklassifiziert & 117 & $15(12,8)$ \\
\hline SDS & 95 & $\mathbf{9}(9,47)$ \\
\hline - SBDS, nicht getestet & 16 & $2(12,5)$ \\
\hline - SBDS-positiv & 78 & $7(8,97)$ \\
\hline - SBDS-negativ & 1 & 0 \\
\hline Zyklische Neutropenie & 98 & $\mathbf{2}(2,04)$ \\
\hline - ELANE-positiv & 56 & $1(1,78)$ \\
\hline - ELANE-negativ & 9 & 0 \\
\hline - ELANE, nicht getestet & 33 & $1(3,03)$ \\
\hline MDS myelodysplastisches Syndrom, SDS Shwachman-Diamond-Syndrom & \\
\hline & &
\end{tabular}

dass die Schwere der Blutbildungsstörung das Leukämierisiko erhöht. Durch eine Folgeerhebung mit Daten bis Dezember 2009 [17] konnte das Leukämierisiko präzisiert werden. Die kumulative Inzidenz nach 15 Jahren beträgt demnach $22 \%$ und ist nicht, wie zuvor vermutet, dramatisch angestiegen. Durch die molekulargenetische Charakterisierung der Patienten ist es inzwischen möglich, das Leukämierisiko mit bestimmten angeborenen Genmutationen zu korrelieren und weitere Risikofaktoren, wie die im Laufe des Lebens erworbenen Mutationen im G-CSF-Rezeptor (CSF3R, [8, 9, 22]) zu identifizieren. Die bis 2017 erhobenen Daten über die Leukämieinzidenz in verschiedenen genetischen Untergruppen aus dem Europäischen Register sind in - Tab. 2 aufgezeigt.

Die Mutationen im CSF3R-Gen treten fast ausschließlich im zytoplasmatischen Teil eines Allels des G-CSF-Rezeptors (Aminosäuren 715-790) auf und führen zum Abbruch der Aminosäurenkette und damit zur stark verlängerten Aktivierung von „signal transducer and activator of transcription 5“ (STAT5, [8, 11, 23]). Die Zeit zwischen dem Erwerb der CSF3R-Mutation und der offenen Leukämie variiert sehr stark und kann mehr als 10 Jahre betragen. Sekundäre leukämie- assoziierte Mutationen beispielsweise im RUNX1-Gen führen dann zusammen mit chromosomalen Veränderungen wie einer Monosomie 7 oder Trisomie 21 zum Anstieg leukämischer Blasten und damit zur offenen AML [22]. Die Leukämie kann nur durch eine allogene Stammzelltransplantation geheilt werden. Das Überleben nach Transplantation beträgt aktuell ca. $80 \%$ [27].

\section{Fazit für die Praxis}

- Das vermehrte Auftreten schwerer bakterieller Infektionen ist häufig ein erster Hinweis auf eine schwere Neutropenie.

- Neutropenien sind mithilfe des Blutbilds einfach zu diagnostizieren; die ursächliche Zuordnung ist häufig schwieriger, dank neuer molekulargenetischer Erkenntnisse aber zunehmend möglich.

- Die Anbindung der Patienten an ein Zentrum und die Registrierung im SCNIR ist bei SCN wichtig, insbesondere da es sich um seltene Erkrankungen handelt, die ein lebenslanges Monitoring erfordern und ein erhöhtes Risiko für sekundäre Leukämien beinhalten.

\section{Korrespondenzadresse}

\section{Prof. Dr. K. Welte}

Pädiatrische Hämatologie und Onkologie, Kinderklinik, Universitätsklinikum Tübingen Hoppe-Seyler-Str. 1, 72076 Tübingen, Deutschland Karl.Welte@med.uni-tuebingen.de

\section{Einhaltung ethischer Richtlinien}

Interessenkonflikt. J. Skokowa, C. Zeidler und K. Welte geben an, dass kein Interessenkonflikt besteht.

Dieser Beitrag beinhaltet keine von den Autoren durchgeführten Studien an Menschen oder Tieren.

Open Access. Dieser Artikel wird unter der Creative Commons Namensnennung 4.0 International Lizenz (http://creativecommons.org/licenses/by/4.0/deed. de) veröffentlicht, welche die Nutzung, Vervielfältigung, Bearbeitung, Verbreitung und Wiedergabe in jeglichem Medium und Format erlaubt, sofern Sie den/die ursprünglichen Autor(en) und die Quelle ordnungsgemäßnennen, einen Linkzur Creative Commons Lizenz beifügen und angeben, ob Änderungen vorgenommen wurden.

\section{Literatur}

1. Andersohn F, Konzen C, Garbe E (2007) Systematic review: agranulocytosis induced by nonchemotherapy drugs. Ann Intern Med 146:657-665

2. Bonilla M, Gillio A, Ruggeiro M et al (1989) Effects of recombinant human granulocyte colonystimulating factor on neutropenia in patients with congenital agranulocytosis. N Engl J Med 320:1574-1580

3. BuxJ, Behrens G, Jaeger G, Welte K(1998) Diagnosis and clinical course of autoimmune neutropenia in infancy: analysis of 240 cases. Blood 91:181-186

4. Cario G et al (2005) Heterogeneous expression pattern of pro- and anti-apoptotic factors in myeloid progenitor cells of patients with severe congenital neutropenia treated with granulocyte colonystimulating factor. Br J Haematol 129:275-278

5. Dale DC, Bolyard AA, Schwinzer BG et al (2006) The severe chronic neutropenia international registry: 10-year follow-up report. Support Cancer Ther 3(4):220-231

6. Dale DC, Person RE, Bolyard AA et al (2000) Mutations in the gene encoding neutrophil elastase in congenital and cyclic neutropenia. Blood 96:2317-2322

7. Dong $\mathrm{F}$ et al (1995) Mutations in the gene for the granulocyte colony-stimulatingfactor feceptor in patients with acute myeloid leukemia preceded by severe congenital neutropenia. N Engl J Med 333:487-493

8. Germeshausen M, Ballmaier M, Welte K (2007) Incidence of CSF3R mutations in severe congenital neutropenia and relevance for leukemogenesis: results of a long-term survey. Blood 109(1):93-99

9. Germeshausen M, Skokowa J, Ballmaier M, Zeidler C, Welte K (2008) G-CSF receptor mutations in patients with congenital neutropenia. Curr Opin Hematol 15:332-337 
10. Gilman PA, Jackson DP, Guild HG (1970) Congenital agranulocytosis: prolonged survival and terminal acute leukemia. Blood 36:576-585

11. Gupta K et al (2014) Bortezomib inhibits STAT5dependent degradation of LEF-1, inducing granulocytic differentiation in congenital neutropenia CD34+ cells. Blood 123:2550-2561

12. Horwitz M, Benson KF, Person RE et al (1999) Mutations in ELA2, encoding neutrophil elastase, define a 21-day biological clock in cyclic haematopoesis. Nat Genet 23:433-436

13. Klein C, Grudzien M, Appaswamy G et al (2007) HAX1 deficiency causes autosomal recessive severe congenital neutropenia (Kostmann disease). Nat Genet 39(1):86-92

14. Kostmann R(1956) Infantile genetic agranulocytosis. Acta Pediatr Scand 45:1-78

15. Makaryan V et al (2015) The diversity of mutations and clinical outcomes for ELANE-associated neutropenia. Curr Opin Hematol 22:3-11

16. Nustede R et al (2016) ELANE mutant-specific activation of different UPR pathways in congenita neutropenia. Br J Haematol 172:219-227

17. Rosenberg PS, Zeidler C, Bolyard AA et al (2010) Stable long-term risk of leukaemia in patients with severe congenital neutropenia maintained on G-CSF therapy. Br J Haematol 150(2):196-199

18. Skokowa J, Welte K (2009) Dysregulation of myeloid-specifictranscription factors in congenital neutropenia. Ann NY Acad Sci 1176:94-100

19. Skokowa J, Cario G, Uenalan M et al (2006) LEF1 is a decisive transcription factor in neutrophil granulopoiesis. Nat Med 12(10):1191

20. Skokowa J, Lan D, Thakur BK et al (2009) NAMPT is essential for the G-CSF-induced myeloid differentiation via a NAD(+)-sirtuin-1-dependent pathway. Nat Med 15(2):151-158

21. Skokowa J, Klimiankou M, Klimenkova $\mathrm{O}$ et al (2012) Interactions among HCLS1, HAX1 and LEF-1 proteins are essential for G-CSF-triggered granulopoiesis. Nat Med 18(10):1550-1559

22. Skokowa J et al (2014) Cooperativity of RUNX1 and CSF3R mutations in severe congenital neutropenia: a unique pathway in myeloid leukemogenesis. Blood 123:2229-2237

23. Skokowa J, Dale DC, Touw IP, Zeidler C, Welte K (2017) Severe congenital neutropenia. Nat Rev Dis Primers. https://doi.org/10.1038/nrdp.2017.32

24. Welte K, Gabrilove J, Bronchud MH, Platzer E, Morstyn G (1996) Filgrastim (r-metHuG-CSF): the first 10 years. Blood 88(6):1907-1929

25. Welte K, Zeidler C, Reiter A et al (1990) Differential effects of granulocyte-macrophage colonystimulating factor and granulocyte colony-stimulating factor in children with severe congenital neutropenia. Blood 75:1056-1063

26. WelteK,ZeidlerC,DaleDC (2006) Severecongenital neutropenia. Semin Hematol 43(3):189-195

27. Zeidler C, Welte K, Barak Y et al (2000) Stem cell transplantation in patients with severe congenital neutropenia without evidence of leukemic transformation. Blood 95:1195-1198

28. Zeidler C, Germeshausen M, Klein C, Welte K (2009) Clinical implications of ELA2-, HAX1-, and G-CSFreceptor (CSF3R) mutations in severe congenital neutropenia. Br JHaematol 144(4):459-467

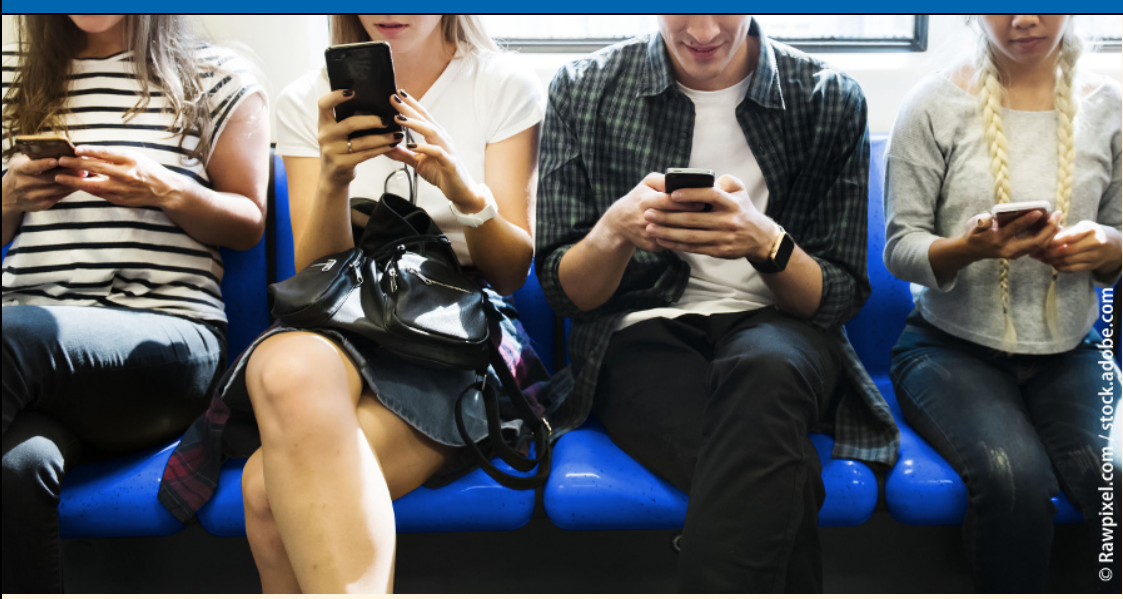

\section{Gesundheitliche und soziale Probleme durch intensiven Gebrauch von sozialen Medien}

\section{WhatsApp, Instagram oder Snapchat können süchtig machen. 2,6 Prozent der Kinder und Jugendlichen erfüllen die Kriterien für eine Abhängigkeit, so die Ergebnisse einer aktuellen Studie.}

Für die Studie „WhatsApp, Instagram und Co. - so süchtig macht Social Media" hat das Forsa-Institut im Auftrag der Krankenkasse DAK-Gesundheit und des Deutschen Zentrums für Suchtfragen am Universitätsklinikum Hamburg-Eppendorf (UKE) 1.001 Kinder und Jugendliche im Alter von zwölf bis 17 Jahren befragt. Erstmals wurde mit dieser Analyse die Häufigkeit einer SocialMedia-Abhängigkeit in einer für Deutschland repräsentativen Stichprobe untersucht. Grundlage sind wissenschaftliche Kriterien aus den Niederlanden (Social Media Disorder Scale).

Kernergebnis der Studie: 2,6 Prozent der Befragten sind bereits süchtig nach Social Media - Mädchen mit 3,4 Prozent etwas häufiger als Jungen (1,9 Prozent). Mädchen sind länger in sozialen Medien unterwegs als Jungen - im Schnitt knapp über drei Stunden pro Tag (Jungen: 2,5 Stunden pro Tag). Je älter die Befragten werden, desto mehr Zeit verbringen sie mit Social MediaAnwendungen: Mädchen zwischen 16 und 17 Jahren sind fast 3,5 Stunden pro Tag in sozialen Medien, gleichaltrige Jungen nur 2,75 Stunden. Je länger und häufiger die Kinder und Jugendlichen online sind, desto höher ist das Suchtrisiko

Laut Untersuchung haben soziale Medien bei den befragten Kindern und Jugendlichen häufig negative soziale Auswirkungen in verschiedenen Bereichen - auch wenn sie nicht als süchtig gelten:

- Jeder dritte Befragte nutzt soziale Medien um nicht an unangenehme Dinge denken zu müssen. Bei den Mädchen trifft dies sogar auf vier von zehn Befragten zu.

- Knapp ein Viertel der Befragten bekommt wegen der Nutzung sozialer Medien manchmal, häufig oder sogar sehr häufig zu wenig Schlaf.

- 22 Prozent streiten manchmal, häufig oder sehr häufig mit den Eltern über die Nutzung sozialer Medien - öfter betroffen sind die 12bis 13-Jährigen (32 Prozent).

- 14 Prozent gaben an, soziale Medien oft heimlich zu nutzen. Ebenso viele können die Nutzung nicht stoppen, obwohl andere ihnen sagten, dass sie dies dringend tun müssen. - 13 Prozent sind unglücklich, wenn sie keine sozialen Medien nutzen können.

- Acht Prozent der Befragten sind mit allen Freunden ausschließlich über soziale Medien in Kontakt.

- Fünf Prozent der Befragten haben regelmäßig kein Interesse mehr an Hobbys oder anderen Beschäftigungen, weil sie lieber Social Media nutzen.

Weitere Informationen für Betroffene, Angehörige und Experten gibt es im Internet unter: www.computersuchthilfe.info

Quelle: DAK-Gesundheit (www.dak.de) 
Hier steht eine Anzeige.

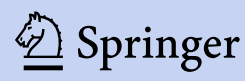

